DOI: 10.30972/eitt.704778

\title{
Variaciones en las características de las aguas del río Negro (Provincia del Chaco, Argentina) desde sus nacientes hasta su desembocadura
}

\author{
Dra. Suárez, Paola A. ${ }^{1}$, Dra. Vega, Marisol ${ }^{2}$, Dra. Vallejos, Silvina ${ }^{3}$
}

\section{Resumen}

Los ríos tienen un rol importante en el desarrollo de múltiples procesos que ocurren sobre la superficie terrestre. A largo de su recorrido, producen reacciones que afectan a su entorno. Así, la interacción del agua de los ríos con el terreno que atraviesan, definen en gran parte las características hidroquímicas y geoquímicas de los cuerpos de agua, que hacen posible o no la vida.

En este trabajo se ha muestreado espacial y temporalmente las variables físicas y químicas de las aguas del río Negro (Provincia del Chaco, Argentina), a fin de evaluar las fuentes que producen sus variaciones: precipitaciones, suelos e influencia antrópica. Se ha evaluado además la influencia de la hidroquímica sobre el comportamiento de los sólidos suspendidos. De esta forma se espera contribuir con el conocimiento sobre la dinámica de las aguas de éste río de llanura y echar luz sobre el efecto de la actividad antrópica sobre ella.

${ }^{1}$ FaCENA (UNNE) - CECOAL (CONICET) | Avenida Libertad 5470 - Laboratorio 33 | paolasuarez792@gmail.com 


\section{Introducción}

Las propiedades del agua de los ríos se encuentran influenciadas por la composición geológica de la cuenca fluvial. De los fenómenos de meteorización y desintegración de rocas en ambientes subacuáticos, una parte de la roca se solubiliza químicamente y otra parte pasa a suspensión en estado coloidal; los elementos en solución en el medio acuoso son precipitados químicamente en función de modificaciones en el pH, Eh y la temperatura (Sabater y Elosegi, 2009).

Además, muchos nutrientes y contaminantes (como los metales pesados y los plaguicidas) se fijan a las partículas finas de sedimento mediante fenómenos de adsorción y, en menor medida, de absorción (Domínguez et al., 2004). De esta forma, los sedimentos influyen sobre la calidad del agua de los ríos, participando como reservorios, medio de transporte y medio de dispersión de esos materiales (Zhu et al., 2008; Horowitz, 1991). Los parámetros más importantes que condicionan los procesos de unión son: el contenido de carbono orgánico, el contenido de arcilla, la humedad, el pH, la capacidad de intercambio catiónico y la temperatura, entre otros (Porta et al., 2003). Por otra parte, las numerosas reacciones químicas y biológicas que ocurren en los cuerpos de agua fluviales pueden modificar las condiciones de $\mathrm{pH}$ y potencial redox del agua, alterando los equilibrios y liberando los elementos contenidos en los sedimentos, los cuales pasan a la columna agua.

En 1963 Livingston realizó un estudio sobre los ríos y lagos más importantes del mundo, donde señalaba que alrededor de 3,9 millones de toneladas de material disuelto eran transportadas por año hacia el mar por estos cuerpos de agua y proporcionó un promedio de concentración (en partes por millón) de los principales constituyentes, entre ellos: bicarbonatos 58,4 , sulfatos 11,2 , cloruros 7,8 , nitratos 1,o, calcio 15,0, magnesio 4,1, sodio 6,3, potasio 2,3, hierro o,67 y sílice 13,1, para un total de 120 ppm.

En la Tabla 1, se recopilan los datos de bibliográficos de composición química para ríos sudamericanos en general, así como también para el río Paraná, curso principal donde el río Negro vierte sus aguas y del cual recibe influencia en los períodos de crecidas. Estos valores promedios se ven afectados por variables climáticas, geográficas, topográficas y edáficas, entre muchas otras. 


\begin{tabular}{|c|c|c|c|c|}
\hline & $\begin{array}{c}\text { Ríos de } \\
\text { Sudamérica } \\
\text { (Livingston, } \\
\text { 1963) }\end{array}$ & $\begin{array}{c}\text { Río Paraná } \\
\text { (Livingston, } \\
\text { 1963) }\end{array}$ & $\begin{array}{c}\text { Ríos de agua } \\
\text { dulce (Webber y } \\
\text { Thurman, 1991) }\end{array}$ & $\begin{array}{c}\text { Río Paraná } \\
\text { (MSAN, OPS, } \\
\text { PNA, UNLP, } \\
\text { 2005) }\end{array}$ \\
\hline Sulfatos & 4,8 & 10 & 12,2 & 7 \\
\hline Cloruros & 4,9 & 15,9 & 7,8 & 13 \\
\hline Nitratos & 0,7 & - & (1) & 0,1 \\
\hline Calcio & 7,2 & 7,3 & 1,5 & 4 \\
\hline Magnesio & 1,5 & 2,8 & 4,1 & 7,6 \\
\hline Sodio & 4 & 15 & 6,3 & 3 \\
\hline Potasio & 2 & 4,1 & 2,3 & 2 \\
\hline Hierro & 1,4 & 2,2 & - & म-1-1 \\
\hline Aluminio & (ב-ב & 1,6 & - & 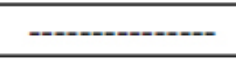 \\
\hline $\begin{array}{l}\text { Alcalinidad } \\
\text { total } \\
\left(\mathrm{mg} \mathrm{CaCO}_{3} / \mathrm{L}\right)\end{array}$ & 25,4 & 28,7 & --1n & 12 \\
\hline Sílice & 11,9 & 20,3 & - & - \\
\hline $\begin{array}{l}\text { Sólidos } \\
\text { Disueltos } \\
\text { Totales/año }\end{array}$ & 69 & 114 & --.+- & 41 \\
\hline
\end{tabular}

Tabla 1. Datos bibliográficos de la composición química de ríos sudamericanos, expresados en ppm.

Por su parte, el agua de lluvia que percola en el suelo ataca a los componentes minerales y libera a las fracciones más solubles, y el agua enriquecida con estos nuevos minerales del suelo llega finalmente a los cuerpos de agua. Pero el aporte de materiales por el agua de escorrentía no es el único camino por el cual la química de los ríos y lagos se ve alterada, también colaboran en éste fenómeno la composición que presente el agua de lluvia y los aportes que realiza el agua subterránea (Ribera y Ordoñez, 2001).

Como hipótesis de este trabajo tenemos que: (1) La composición química de este río de llanura se encuentra fuertemente influenciada por la estacionalidad climática. (2) El contenido de compuestos mayoritarios presentes en la fase líquida del sistema fluvial, varía longitudinalmente en relación con las características de los materiales que ingresan a la cuenca debido principalmente a la acción antrópica.

Por lo tanto el objetivo principal de este trabajo ha sido evaluar las características tanto física como químicamente la columna de agua, determinando en ésta tanto 
los sólidos en suspensión como las variables químicas mayoritarias a lo largo del recorrido del río Negro.

\section{Área de estudio}

El río Negro, localizado en la provincia del Chaco (República Argentina) y pertenece a la cuenca del Plata. Es un ejemplo característico de áreas con escasa pendiente, incluidos dentro de los Sistemas Hidrológicos No Típicos. Es un curso fluvial de llanura subtropical que, en un recorrido relativamente corto, recibe aportes de salinidad por los suelos, influencia antrópica en diferentes escalas y presenta variaciones climáticas marcadas entre los períodos de estiaje y períodos húmedos. Además, la cuenca de este río es considerada de importancia local por ser uno de los principales tributarios del río Paraná, y de importancia internacional por formar parte de humedales protegidos para su conservación y uso racional.

Cuatro puntos de muestreo fueron elegidos, cuya ubicación se muestra en la Figura 2 y en la Figura 3, y los cuales son representativos de los siguientes tramos fluviales: (P1) área de cabeceras con bajo caudal, baja influencia antrópica y abundante vegetación. (P2) y (P3) tramo medio encauzado, con mayor caudal que el encontrado en el punto 1, baja ocupación humana y abundante vegetación durante la mayor parte del año. En el punto 3 existe una obra de regulación que desvía el escurrimiento aguas abajo hacia un cauce cercano, durante las épocas lluviosas, a fin de evitar las inundaciones de las zonas pobladas. Y (P4) tramo distal cercano a su desembocadura, ubicado aguas debajo de la zona urbana, con más de dos veces el caudal encontrado en los puntos anteriores, alto impacto antrópico por vertido de efluentes domésticos e industriales (industria frigorífica y láctea) y donde la abundancia de la población vegetal varía a lo largo del año. Las coordenadas geográficas correspondientes a cada punto de muestreo pueden consultarse en la Tabla 2.

Pese a su origen autóctono, en su tramo bajo el río Negro recibe una fuerte influencia del régimen de crecidas del río Paraná, su colector final, el cual durante crecidas extremas produce un importante retardo del escurrimiento que afecta de modo directo la dinámica hidrosedimentológica del tributario (Poi de Neiff et al., 2004); conjuntamente, recibe un importante aporte de las aguas subterráneas en toda su cuenca. 


\begin{tabular}{|c|c|}
\hline Punto de Muestreo & Coordenadas geográficas \\
\hline P1 - Localidad “Colonia Elisa" & $27^{\circ} 04^{\prime} 32^{\prime \prime} \mathrm{S} 59^{\circ} 27^{\prime} 23^{\prime \prime} \mathrm{W}$ \\
\hline P2 - Localidad “La Verde" & $27^{\circ} 08^{\prime} 40,5^{\prime \prime} \mathrm{S} 59^{\circ} 23^{\prime} 38,8^{\prime \prime} \mathrm{W}$ \\
\hline P3 - Localidad “Laguna Blanca" & $27^{\circ} 13^{\prime} 6,4^{\prime \prime} \mathrm{S} 59^{\circ} 13^{\prime} 15,1^{\prime \prime} \mathrm{W}$ \\
\hline P4 - Localidad "Resistencia" & $27^{\circ} 25^{\prime} 49^{\prime \prime} \mathrm{S} 58^{\circ} 56^{\prime} 57.7^{\prime \prime} \mathrm{W}$ \\
\hline
\end{tabular}

Tabla 2. Coordenadas geográficas de los puntos de muestreo.

El área del río Negro estudiada en este trabajo se encuentra incluida dentro de las 508.00o ha del Este chaqueño que fueran designadas en 2004 como sitio RAMSAR con el no 1366 (RAMSAR, 2013).

El río Negro se encuentra expuesto a un clima subtropical atlántico húmedo, con un régimen pluviométrico anual muy variable que condiciona el estado hidrológico de los sistemas fluviales regionales. Los picos de máximas precipitaciones, que ocurren entre los meses de noviembre y marzo, representan cerca del 80-90\% de las precipitaciones anuales, mientras que los picos mínimos tienen lugar entre julio y agosto (Morello, 1983). Las precipitaciones anuales se caracterizan por una disminución de este a oeste lo que da lugar a que la cuenca del río Negro quede comprendida entre la isohieta de $900 \mathrm{~mm} 3$ en el extremo oeste y $1300 \mathrm{~mm} 3$ al este, cerca de su desembocadura.

El registro de precipitaciones (Administración Provincial del Agua, ente perteneciente a la Provincia del Chaco, Argentina - APA-) deja en evidencia el período hiperseco de la región durante la mayoría de los períodos muestreados. Cabe destacar que unos diez años atrás los tramos urbanizados del río Negro, que atravesaban la Ciudad de Resistencia (Chaco), generaron grandes perjuicios a la población, por lo que se realizó una desviación de su caudal a la altura de su tramo medio para conducir los excedentes hídricos a una cuenca vecina. 


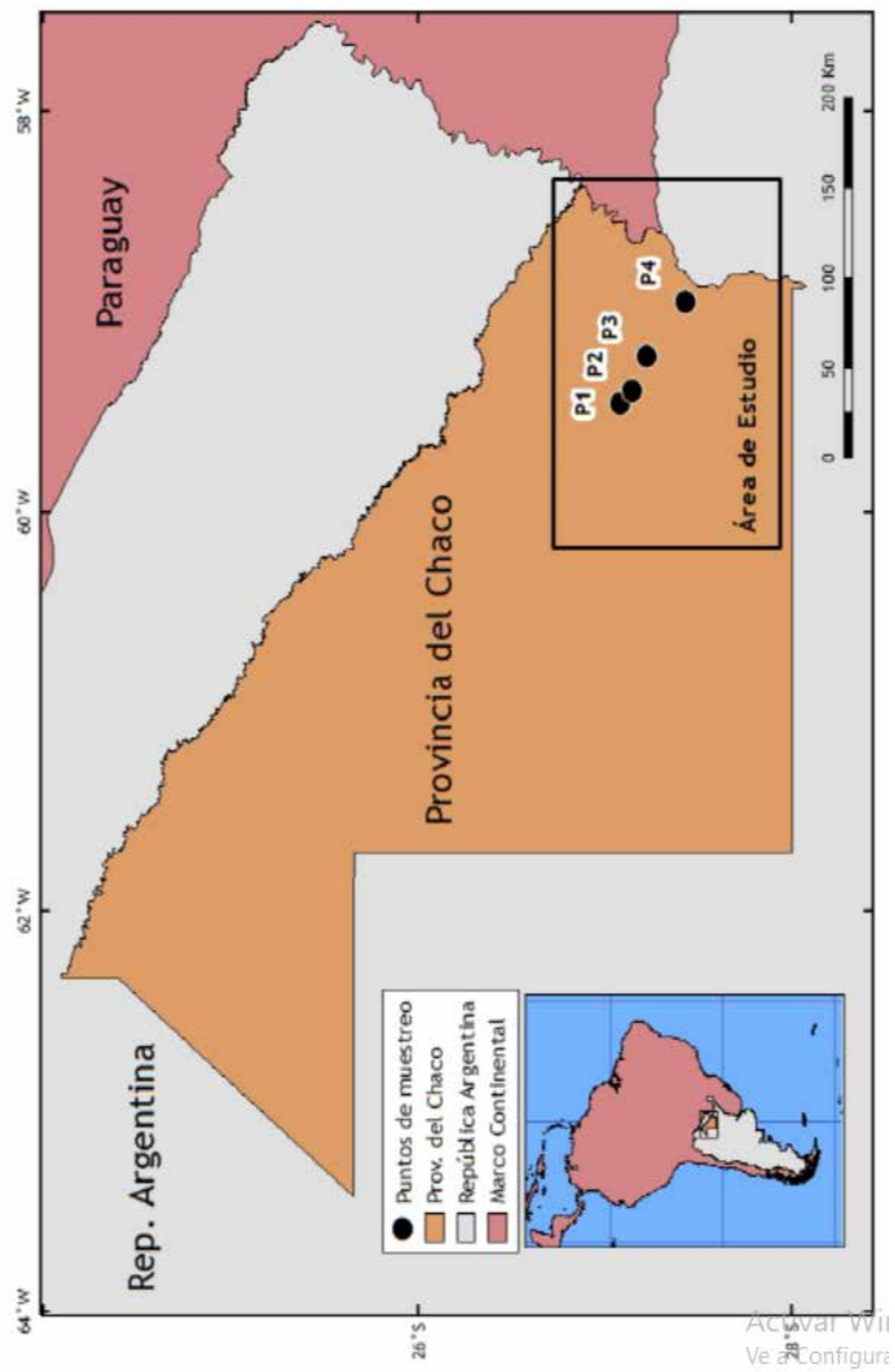

Figura 2. Mapa de los puntos de muestreo. Proyección 1:100.0oo.ooo. 


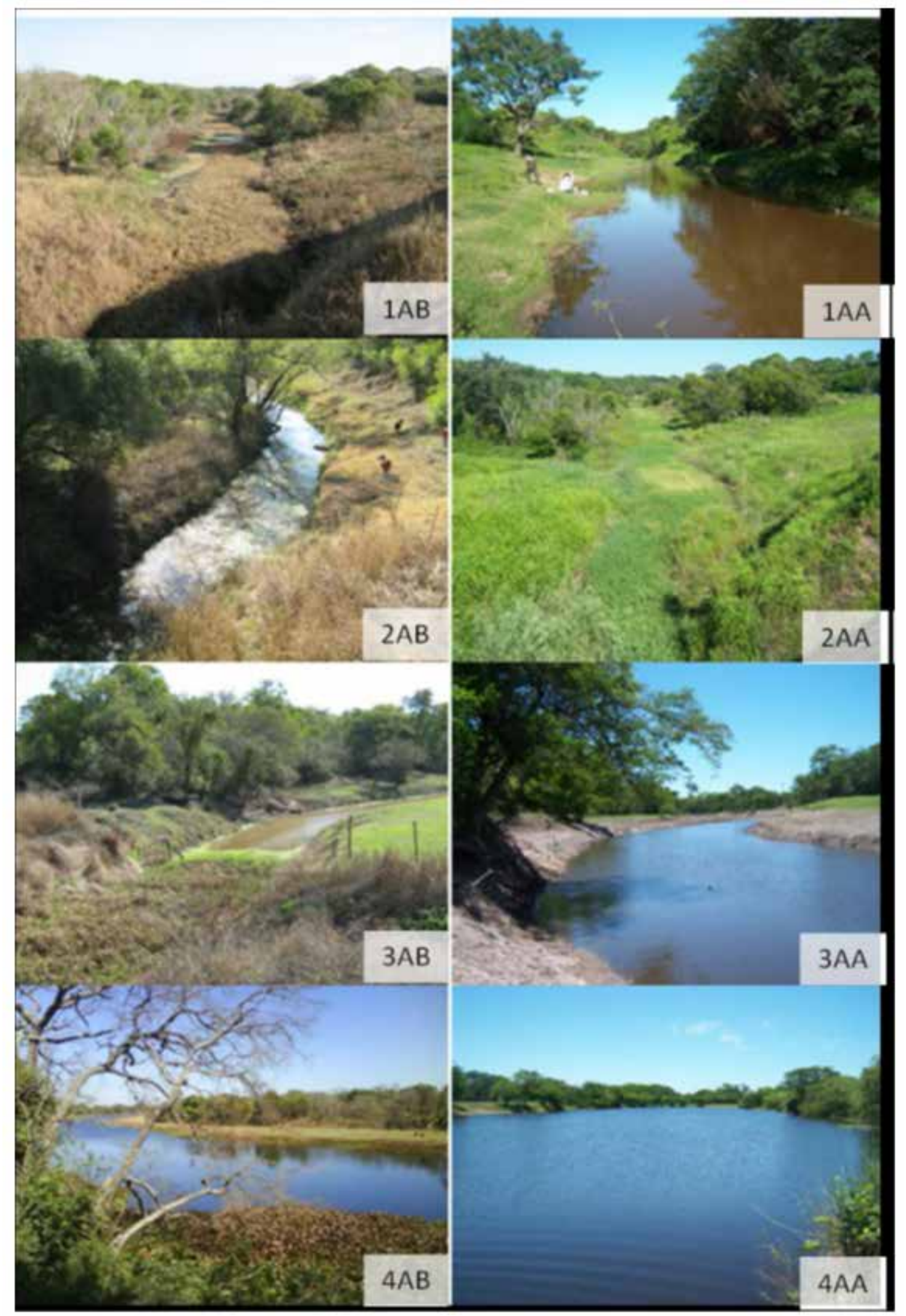

Figura 3. Puntos de muestreo (1 a 4), en período de aguas altas ( $A A$ ) y aguas bajas ( $A B)$. 
En el área de estudio, las aguas subterráneas salobres y el contenido salino propio de los suelos, junto con el déficit hídrico que ocasionalmente sufre la región, ha generado la presencia de suelos salinos y salino-alcalinos, con alta proporción de sodio combinado con sulfato y cloruro, que en el complejo de intercambio supera el 15\% (OEA, 1975; Ledesma y Zurita, 1995). Los albardones y las áreas inundables también se encuentran condicionados por la salinidad y la sodicidad.

En la Figura 4 se presenta la distribución de los suelos en el área de muestreo y las zonas cercanas. Se observa que los cuatro puntos de muestreo de este trabajo pasan por suelos del orden molisol. Cabe destacar, sin embargo, que aguas arriba de las áreas de nacientes los suelos son del orden entisol. Debido a que el material proveniente del lavado de los suelos superficiales se transporta en solución hacia el cauce de los ríos cercanos, puede ocurrir que el punto de muestreo 1 presente características hidrogeoquímicas algo diferentes a los demás puntos de muestreo.

Smith (1986) describe a los suelos del orden entisol como suelos jóvenes, a veces poco o nada desarrollados, que se caracterizan por su naturaleza mineral. Por su parte, los suelos del orden molisol son ricos en materia orgánica y en iones alcalinos con dominancia $\mathrm{Ca}++\mathrm{y} \mathrm{Mg}++$, siendo frecuente encontrar horizontes ricos en acumulación de arcilla que pueden estar formando agregados, así como horizontes ricos en carbonatos cálcico-magnésicos (15\% o más) y horizontes nátricos (con más del $15 \%$ de $\mathrm{Na}+$ intercambiable).

La salinidad actúa en los suelos de la región como un factor que acrecienta la inestabilidad estructural propia de estos suelos, constituidos por materiales aluviales poco consolidados, escasamente permeables y dominados por la fracción pelítica (Neiff, 1986; Patiño y Orfeo, 1986), aumentando el riesgo a la erosión hídrica en esta región.

\section{Materiales y métodos}

Durante un período de tres años se han realizado muestreos estacionales en los cuatro puntos de muestreo mencionados anteriormente, teniendo en cuenta el ciclo hidrológico, a fin de obtener registro de los períodos húmedos (aguas altas) y de estiaje (aguas bajas), coincidentes con las estaciones de verano e invierno australes respectivamente, resultando en consecuencia seis muestreos en total. 


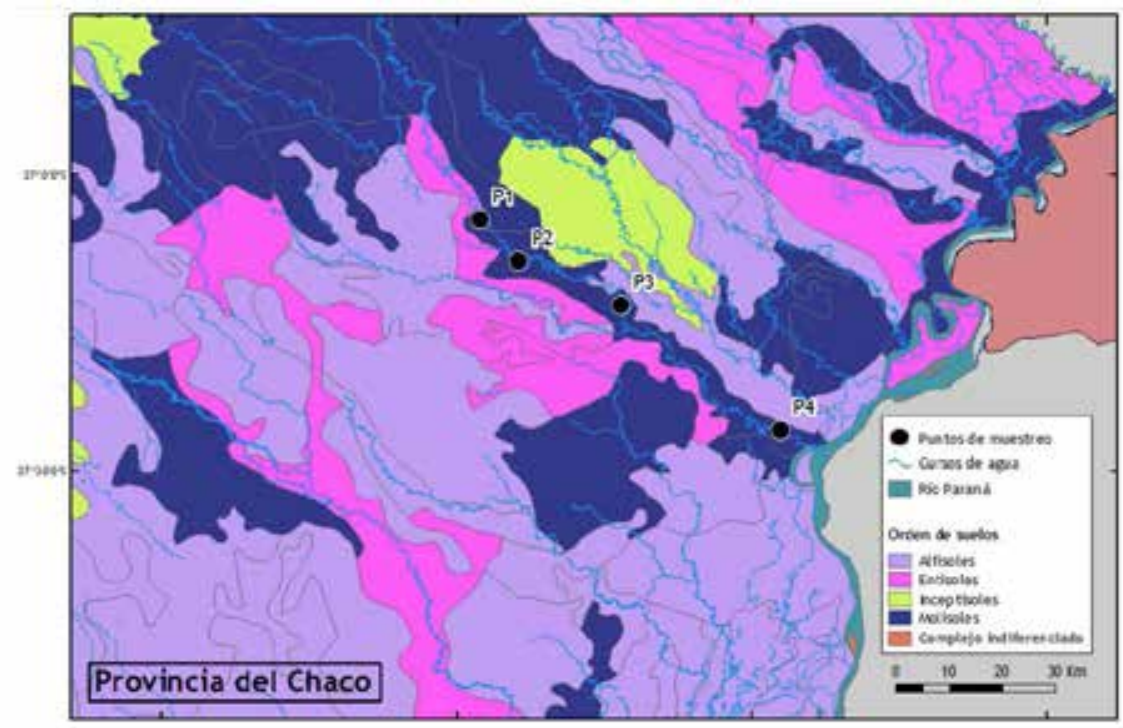

Figura 4. Mapa de suelos de la región. Proyección 1:80o.ooo

Para la toma de muestras se utilizó botella de Van Dorn de dos litros de capacidad (Figura 5) para extraer muestras de agua del centro del cauce, las cuales posteriormente se fraccionaron en tres submuestras y se guardaron en botellas de PET. Una de ellas se cerró con aire remanente, para posterior análisis de materiales suspendidos, una segunda botella cerrada herméticamente, sin aire y refrigerada, para las determinaciones químicas, y una tercera botella para ser conservada como réplica de control. Las variables hídricas de temperatura, oxígeno disuelto, conductividad eléctrica y $\mathrm{pH}$ se midieron in situ.

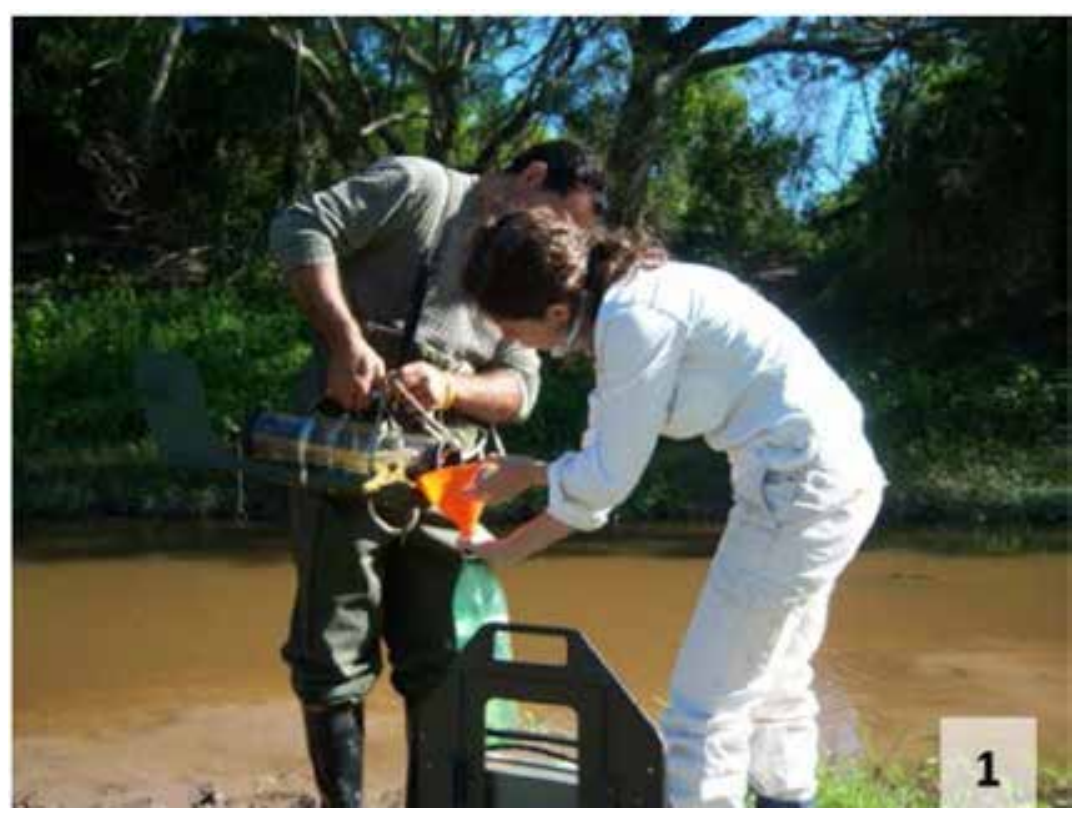

Figura 5. Material de muestreo, botella de Van Dorn 


\section{Resultados y discusiones}

Se ha utilizado la información obtenida en seis campañas de muestreo para obtener los parámetros estadísticos de las variables correspondientes a la composición mayoritaria del agua y sus principales parámetros físico-químicos. De la observación de la tabla de estadísticos por variable (Tabla 3) y de los gráficos univariantes, pueden extraerse algunas conclusiones preliminares: (a) los cloruros, sulfatos y sodio han presentado los valores medios más altos, por lo que son dominantes en la composición química del agua del río Negro; (b) el catión Mg es el que se ha encontrado en menores concentraciones, seguido por el potasio y el calcio; (c) la acidez del agua, expresada como $\mathrm{pH}$, tuvo un comportamiento homogéneo a lo largo de toda la cuenca, con valores que oscilaron alrededor de un valor cercano a la neutralidad, lo cual coincide con la información disponible en trabajos anteriores (Lancelle et al., 1986; COFEA, 1995; MSAN, OPS, PNA, UNLP, 2005); (d) los valores de alcalinidad determinan que las aguas del río Negro se encuentran en un rango medio de productividad; (e) la mayoría de las variables determinadas para aguas presentan una distribución asimétrica; sin embargo $\mathrm{pH}$, potasio y alcalinidad han sido los que menor asimetría presenta la distribución de sus valores; (f) la prueba de normalidad de Kolmogorov-Smirnov muestra que la gran mayoría las variables consideradas tienen una distribución no normal, con un valor de la probabilidad ( $\mathrm{p}$ a posteriori) menor a 0,05 . Únicamente el $\mathrm{pH}$ y la alcalinidad siguen una distribución normal ( $\mathrm{p}=0,113$ y $\mathrm{p}=0,085$ respectivamente); (g) los diagramas Box-plot muestran la presencia de valores anómalos (outliers) para todas las variables, a excepción del $\mathrm{pH}$, lo cual indica valores extremos debido posiblemente a las variaciones del caudal entre períodos lluviosos y de estiaje.

Si bien los valores obtenidos para algunas concentraciones iónicas del río Negro son altos con respecto a los citados por Livingstone (1963) para los ríos sudamericanos, bibliografía más reciente indica que siguen un patrón común a la composición de los ríos locales (Lancelle et al., 1986; COFEA, 1995; Bonetto et al., 1998; Villar y Bonetto, 2000; MSAN, OPS, PNA, UNLP, 2005; Depetris y Pasquini, 2007). La MSAN, OPS, PNA, UNLP (2005) indicó para los ríos de la zona un orden de abundancia de: $\mathrm{Ca} 2+>\mathrm{Mg} 2+>\mathrm{Na}+>\mathrm{K}+$ en algunos sectores, $\mathrm{y}(\mathrm{Na}+\mathrm{K}+)>(-$ Ca2+Mg2+) donde la concentración de sólidos disueltos totales aumenta. Depetris y Pasquini (2007) señalaron para el río Paraná (colector final del río Negro), 
un orden de abundancia para los aniones mayoritarios de $\mathrm{HCO}_{3}->\mathrm{Cl}->\mathrm{SO}_{42}-$, y para los cationes mayoritarios de $(\mathrm{Na}+\mathrm{K}+)>\mathrm{Ca} 2+>\mathrm{Mg} 2+$. Para el río Negro hemos determinado que el orden de abundancia es similar, para aniones mayoritarios es $\mathrm{HCO}_{3}->\mathrm{SO} 42->\mathrm{Cl}-$, y para cationes mayoritarios $\mathrm{Na}+>\mathrm{K}+>\mathrm{Ca} 2+>\mathrm{Mg} 2+$. La concentración del ión potasio es significativamente más alta que la registrada en los trabajos mencionados anteriormente; sin embargo, en la misma bibliografía se hace referencia a que esto sucede en períodos excepcionales. La concentración de K registrada en el río Negro se corresponde con los valores mencionados por APHA (1998) para aguas saladas.

En la Figura 6 se representan los datos hidroquímicos de todas las muestras de agua recolectadas en los cuatro puntos de muestreo, durante las seis campañas realizadas en el desarrollo de este trabajo. Las muestras que se presentan en dicho gráfico han sido etiquetadas mediante un código $\mathrm{X}-\mathrm{Y}$, donde $\mathrm{X}$ indica el número de campaña de muestreo (1 a 6) e Y simboliza el punto de muestreo (1 a 4). De acuerdo a la clasificación química de Piper (1944), la concentración de aniones y cationes mayoritarios permiten determinar que las aguas del río Negro son principalmente bicarbonatadas sódico-potásicas. Se observan variaciones composicionales importantes en dos de las campañas pertenecientes a períodos de aguas bajas ( 1 y 5), indicando procesos de concentración de iones en ciertos puntos de muestreo.

\begin{tabular}{|c|c|c|c|c|c|c|c|c|c|c|}
\cline { 2 - 10 } \multicolumn{1}{c|}{} & $\mathbf{p H}$ & Conduct. & Alcal. & $\mathbf{C l}$ & $\mathbf{S O}_{4}$ & $\mathrm{Ca}$ & $\mathrm{Mg}$ & $\mathrm{Na}$ & $\mathrm{K}$ & CSS \\
\cline { 2 - 10 } & & $\mu \mathrm{S} / \mathrm{cm}$ & $\mathrm{mgCaCO} / \mathrm{L}$ & $\mathrm{mg} / \mathrm{L}$ & $\mathrm{mg} / \mathrm{L}$ & $\mathrm{mg} / \mathrm{L}$ & $\mathrm{mg} / \mathrm{L}$ & $\mathrm{mg} / \mathrm{L}$ & $\mathrm{mg} / \mathrm{L}$ & $\mathrm{mg} / \mathrm{L}$ \\
\hline Media & 6,83 & 474 & 111 & 45 & 50 & 17,3 & 8,8 & 52,9 & 22,5 & 108,5 \\
\hline Mediana & 6,70 & 262 & 105 & 17 & 21 & 13,2 & 7,0 & 26,4 & 17,9 & 64,3 \\
\hline Desv. estándar & 0,57 & 561 & 60 & 108 & 96 & 11,9 & 8,5 & 97,5 & 10,1 & 166,1 \\
\hline Curtosis & $-1,40$ & 15 & 2 & 22 & 19 & 1,7 & 11,3 & 19,8 & 1,1 & 15,3 \\
\hline Coef. asimetría & 0,21 & 4 & 1 & 5 & 4 & 1,4 & 3,0 & 4,3 & 1,5 & 3,7 \\
\hline Minimo & 6,05 & 88 & 29 & 4 & 2 & 3,7 & 2,0 & 0,4 & 12,2 & 10 \\
\hline Máximo & 7,80 & 2870 & 287 & 540 & 480 & 52,0 & 43,0 & 492 & 48,5 & 815 \\
\hline N2 de muestras & 24 & 24 & 24 & 24 & 24 & 24 & 24 & 24 & 24 & 24 \\
\hline
\end{tabular}

Tabla 3. Resumen estadístico de los datos los parámetros medidos en las aguas del río Negro. 
En la Figura 7 se presenta el diagrama de Stiff, donde es posible observar la variación en la composición química del agua, desde las nacientes (1) hasta las áreas de desembocadura (4). De la gráfica puede deducirse que los valores de calcio se mantienen casi constantes en los tres primeros puntos de muestreo, mientras que junto con los iones de cloruro, sulfato, magnesio y sodio aumentan notoriamente en el punto de muestreo 4, relacionado principalmente con el vertido de desechos urbanos y la actividad antrópica de la tierra aguas arriba.

Para determinar si las variaciones composicionales del agua se encuentran mayormente dominadas por factores espaciales o estacionales, se han confeccionado diagramas de Piper para cada punto de muestreo (Figura 8). La composición catiónica, principalmente sódico-potásica, es por lo general homogénea, tanto estacional como longitudinalmente, evolucionando ligeramente a magnésico-cálcica en los períodos húmedos. Esta variación indica la ocurrencia de procesos de disolución en los suelos de la cuenca durante los meses lluviosos. La composición aniónica bicarbonatada se mantiene en todas las estaciones de muestreo. Se observa nuevamente un aumento importante de cloruros y moderado de sulfatos, acentuado en las épocas de estiaje, en el punto de muestreo 4. Se puede decir entonces que la hidroquímica del punto de muestreo 4 es la responsable de los valores irregulares observados en la Figura 6, resultante del lavado de los suelos superficiales ricos en estos iones, pero fundamentalmente a consecuencia de la actividad antrópica aguas arriba, lo cual se refleja en el cambio brusco que experimenta la composición química del agua en este sector de la cuenca.

La composición química del agua de los ríos no sólo está influenciada por la constitución original de las rocas y los minerales del suelo por donde drenan, el tiempo de estadía del agua también es determinante. Al considerar el contexto de llanuras donde se enmarca el río Negro, se reconoce la existencia de un drenaje lento, que en ocasiones es interrumpido por la abundante vegetación palustre (Neiff y Orfeo, 2003). Estos procesos favorecen un activo intercambio iónico en la interfase agua-sedimento durante los prolongados períodos de estancamiento del agua, siendo posiblemente esta la razón por la cual el punto de muestreo situado en las aéreas de nacientes del río presenta concentraciones iónicas más elevadas, superadas únicamente por las aéreas de desembocadura. 
La Figura 9 muestra la variación de la conductividad eléctrica del agua (CEA) y su relación con la concentración de sólidos suspendidos (CSS). En la gráfica se indican con color ocre los muestreos realizados durante períodos de estiajes y con color celeste los correspondientes a períodos lluviosos. Se observa una variación inversa entre ambas variables, regulada por la estacionalidad, lo cual también ha sido observado por otros autores para ríos de la zona (Patiño y Orfeo, 1997; Suárez et al., 2010). Según Orfeo (1999), durante los períodos de aguas bajas es frecuente que la CSS de los ríos aumente; sin embargo, destaca que en algunos cursos (especialmente los de composición hidroquímica predominantemente salina), esta tendencia se invierte. Durante los períodos de estiaje la concentración de sales aumenta, lo cual favorece la aglutinación de materiales finos y acelera los procesos deposicionales. Sin embargo, durante los períodos lluviosos el elevado caudal diluye las sales, y disminuye la CSS.

Las concentraciones de sólidos en suspensión más bajas se han registrado en los puntos de muestreo 1 y 4 (nacientes y desembocadura, respectivamente). En el punto 1 las bajas concentraciones parecen estar relacionadas con una elevada concentración de iones y de sedimentos (ambos factores favorecen la floculación y la consecuente aceleración de la sedimentación), debido al bajo caudal. En el punto 4 las concentraciones iónicas parecen estar relacionadas principalmente con la actividad antrópica aguas arriba, así como con la incorporación de sales proveniente del lavado de los suelos, que aumenta la CEA. Sin embargo, la disminución de la CSS en este último punto también se encuentra relacionada con procesos de dilución.

Durante la campaña 5 se registra una alta concentración de sedimentos suspendidos en todos los puntos de muestreo. Este suceso puede deberse a concentración de materiales, como se ha mencionado anteriormente, ya que corresponde a una campaña realizada durante período de estiaje; sin embargo, este comportamiento homogéneo en todos los puntos de muestreo podría ser consecuencia de precipitaciones. Durante los períodos de estiaje, el caudal del río es tan bajo que alteraciones como las generadas por las gotas de lluvia pueden agitar sus aguas, con la consecuente puesta en suspensión de los materiales finos que se encuentran depositados en el fondo del cauce. El lavado superficial de los suelos durante estos eventos pluviométricos también aumenta la concentración de materiales en suspensión. 
Algunos autores como Bielsa y Fratti (1981), Lancelle et al., (1986), Patiño y Orfeo (1997), Ruberto (1999), Poi de Neiff (2004) y Suárez et al. (2010), han destacado el efecto que produce la pluviometría sobre las características químicas del agua de los ríos y el transporte de los sedimentos en otros ríos de la región.

En la Figura 10 se presenta el registro histórico de las precipitaciones para cada uno de los puntos de muestreo seleccionados los cuales han sido proporcionados por la Administración Provincial del Agua, ente perteneciente a la Provincia del Chaco, República Argentina (APA, 2010).

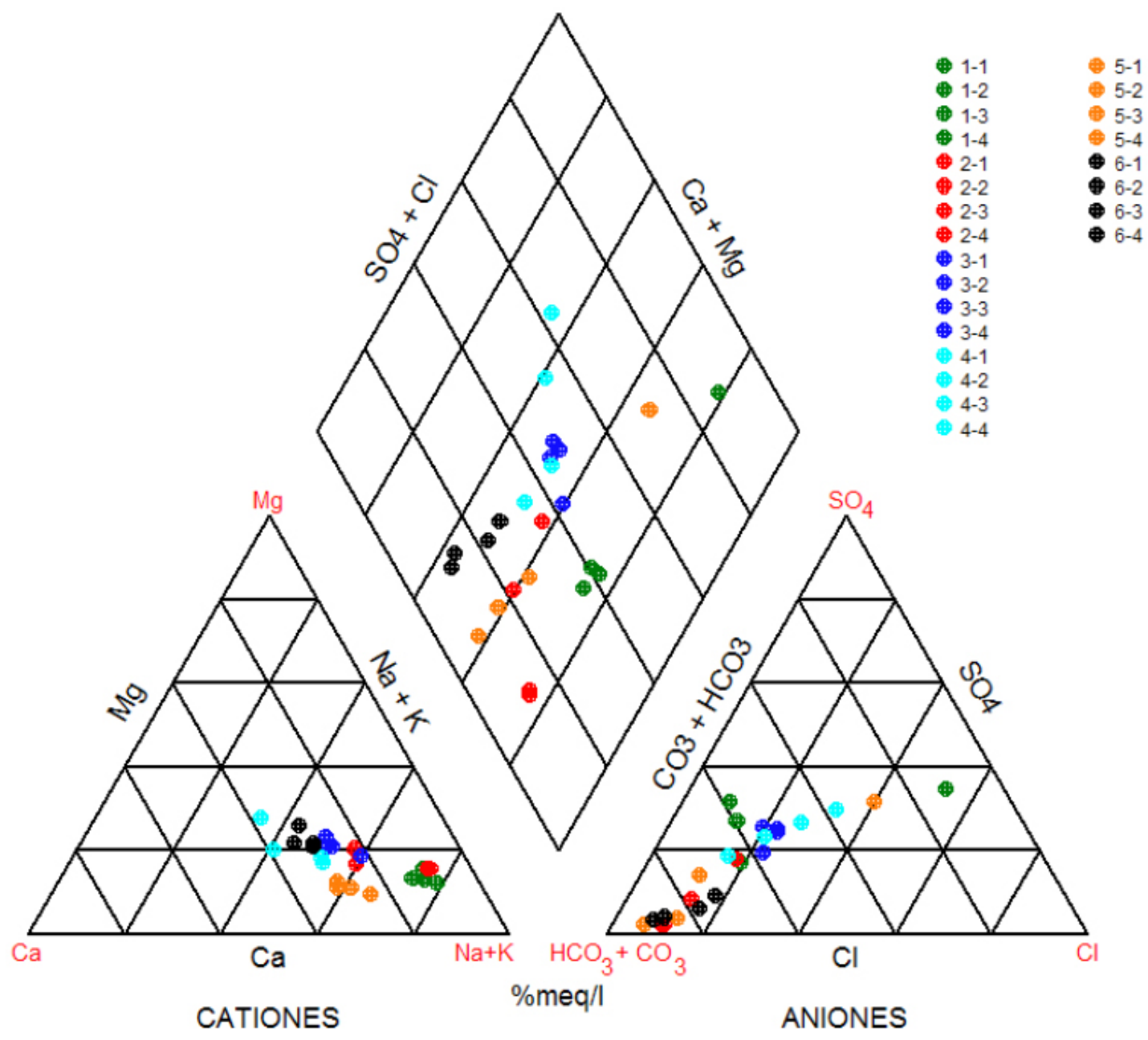

Figura 6. Diagrama de Piper. Referencias: Campaña-Punto de muestreo. 


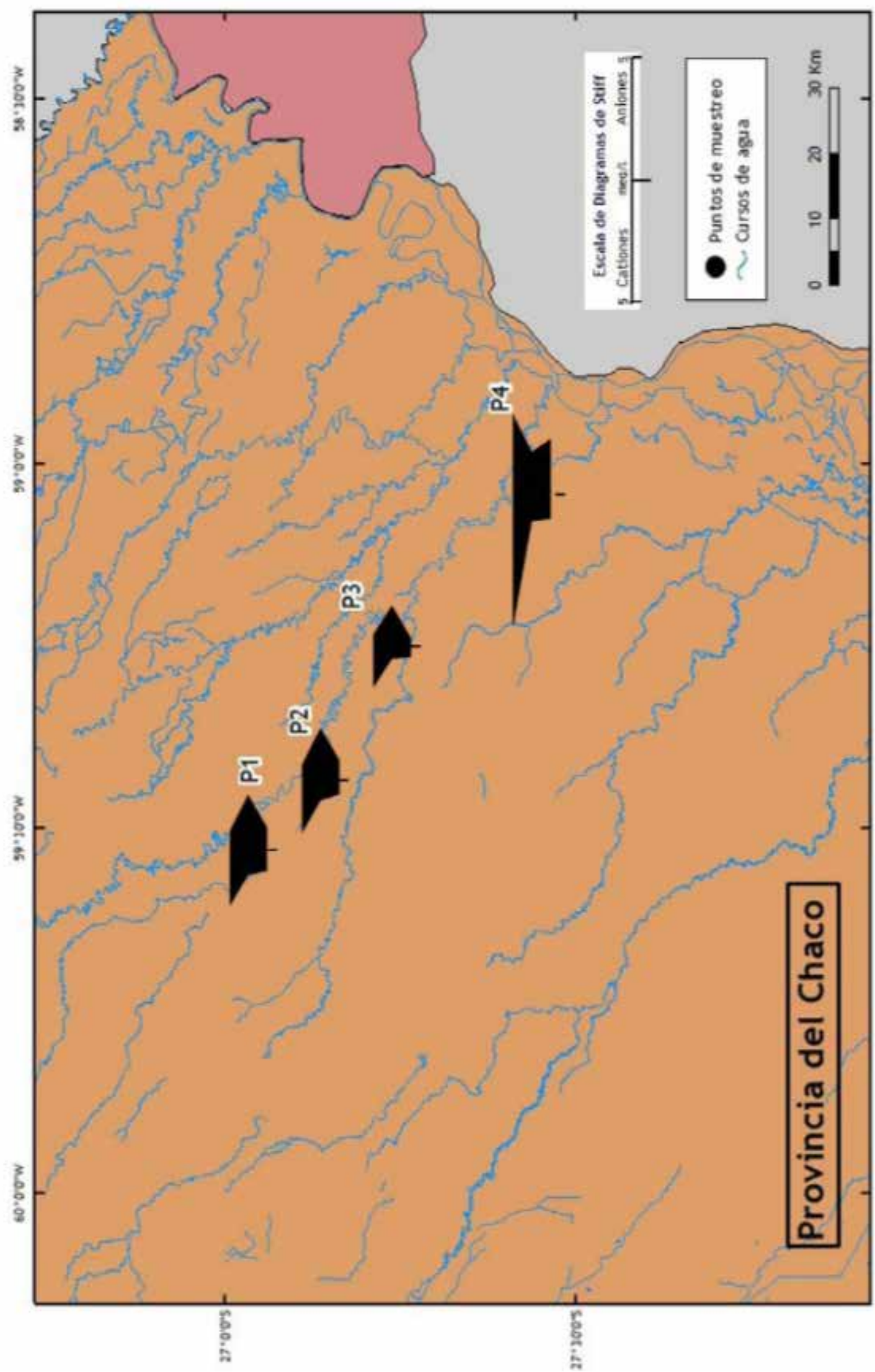

Figura 7. Representación de la variación longitudinal de las características hidroquímicas mediante diagramas de Stiff. 


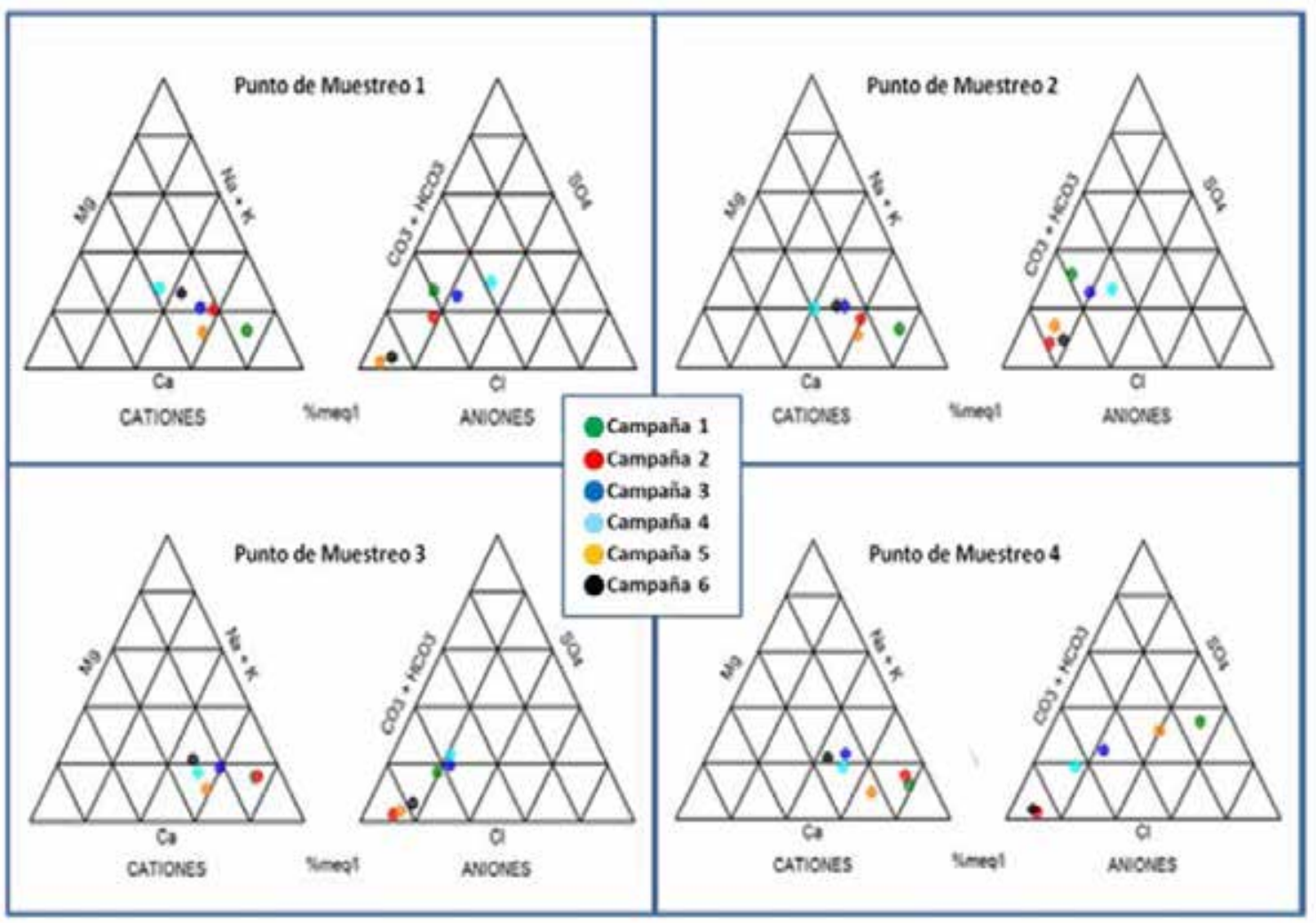

Figura 8. Diagrama de Piper para cada estación de muestreo.

Se observa que las precipitaciones caídas durante el año 2008 son notoriamente menores que las registradas en los años posteriores, es por ello que pueden acentuarse los procesos de concentración iónica y bajo transporte de sedimentos. En el año 2010, sin embargo, las precipitaciones fueron muy superiores, tanto en período de estiaje como durante el período lluvioso, por esta razón procesos de dilución, lavado de suelos superficiales y transporte de sedimentos, entre otros, pueden verse aumentados. La amplia variación en la pluviometría de la región, así como sus efectos en la dinámica fluvial, ya ha sido advertida por otros autores (Morello, 1983; Patiño y Orfeo, 1986).

\section{Conclusiones}

Las aguas superficiales analizadas del río Negro son principalmente bicarbonatadas sódicas, con un orden de abundancia relativa de $\mathrm{HCO}_{3}->\mathrm{SO} 42->\mathrm{Cl}-$ para los aniones mayoritarios y $\mathrm{Na}+>\mathrm{K}+>\mathrm{Ca} 2+>\mathrm{Mg} 2+$ para la composición catiónica. Las variaciones composicionales se encuentran controladas en parte por un factor espacial, aunque el factor temporal ha resultado ser el más significativo. 


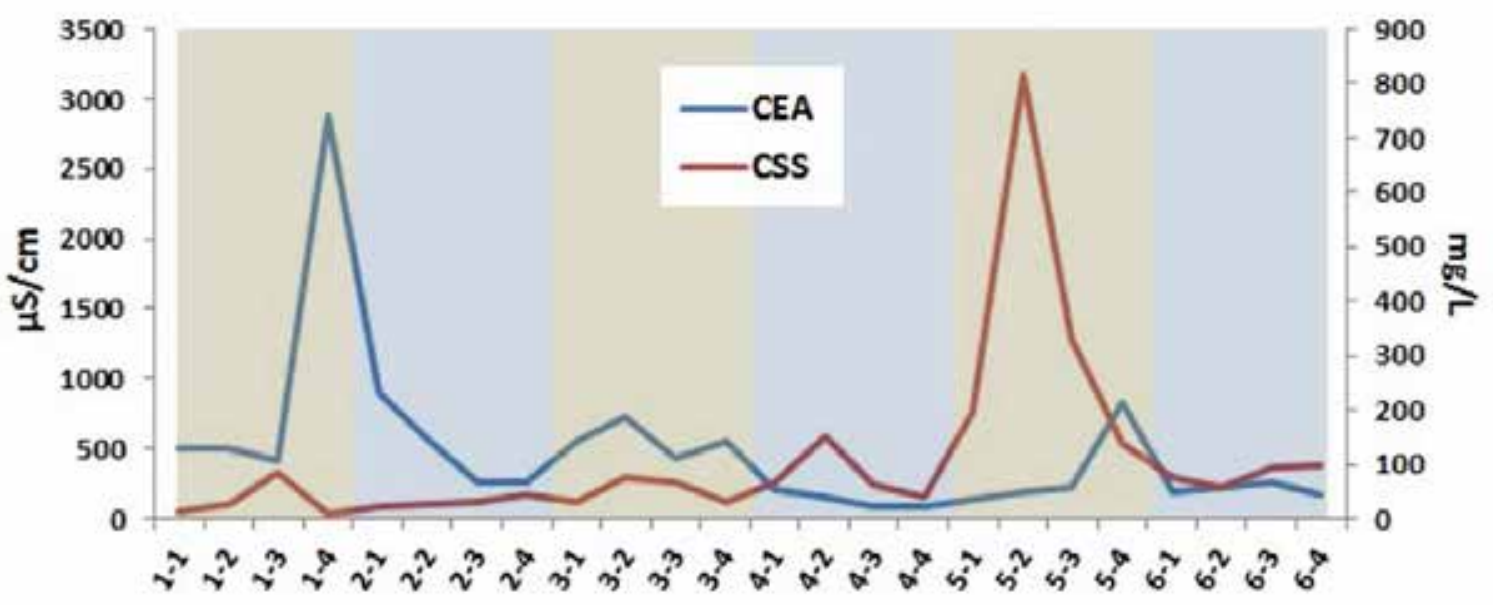

Figura 9. Variación longitudinal de CEA y CSS. El primer número denomina la campaña y el segundo el punto de muestreo. En ocre las campañas de aguas bajas y en celeste las campañas de aguas altas.

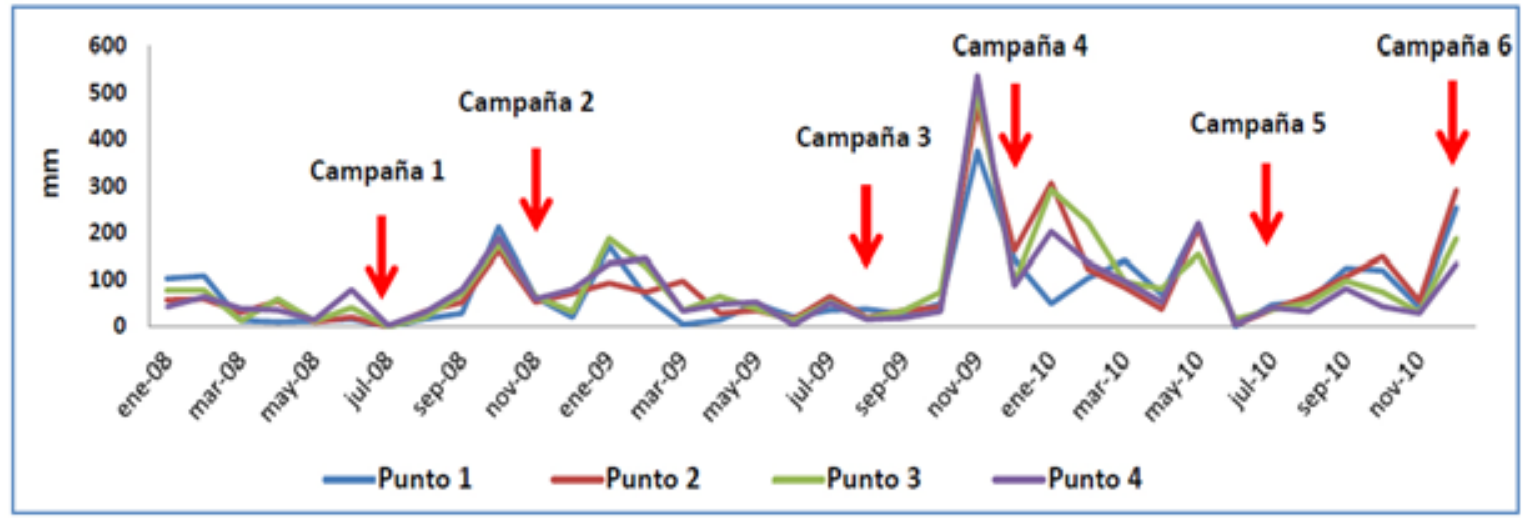

Figura 10. Registro histórico de precipitaciones en el área de muestreo. Las flechas en rojo indican los meses en que fueron tomadas las muestras.

La composición de las aguas del río Negro indica concentraciones más elevadas de las variables químicas estudiadas en los períodos de estiaje y en relación al punto de muestreo 4, cercano a la desembocadura.

El factor espacial en todos los casos se encuentra determinado por el punto de muestreo cercano al área de desembocadura del sistema fluvial, punto 4, donde existe mayores concentraciones de las variables químicas estudiadas, lo cual se encuentra relacionado con la acción antrópica por vertidos domésticos e industriales. Aunque también el lavado de los suelos superficiales tiene un rol importante en las variaciones observadas. 
La concentración de sólidos en suspensión estuvo influenciada inversamente por la concentración de las variables químicas estudiadas, así como por las precipitaciones ocurridas.

\section{Bibliografía}

APA (2010) Administración Provincial del Agua, Provincia del Chaco (Argentina) (último acceso 21/11/2013)

http://www.ecomchaco.com.ar/apa/institucional/eb/hidrometeorologia.htm

APHA, AWWA, WEF (1998) Standard methods for examination of water and wastewater, American Public Health Association, Washington D. C.

Bielsa, L.; Fratti, R. (1981) Determinación del comportamiento del sistema natural y modificado con obras en temas referentes a la calidad del agua. CFI, Programa Bajos Submeridionales, Santa Fe, 42 pp.

Bonetto, C.; Villar, C. A.; de Cabo, L.; Vaithiyanathan, P. (1998) Hydrochemistry of a large floodplain river. Verh. Internat. Verein. Limnol., 26, 899- 902pp.

COFEA (1995) Contaminación en cursos de agua del Chaco oriental. Report: 148pp.

Depetris, P. J.; Pasquini, A. I. (2007) The Geochemistry of the Paraná River: An Overview. In The Middle Paraná River: Limnology of a Subtropical Wetland: 6: 143-174pp.

Domínguez-Chicas, A.; Kretzschmar, T.; Núñez-Sánchez, F. (2004) Velocidades de sedimentación en aguas pluviales de Cd. Juárez, Chihuahua, México. Revista Mexicana de Ciencias Geológicas. Vol. 21 (3), 412-420pp.

Horowitz, A.J. (1991) A primer on trace metal-sediment chemistry. 2da Edic. U.S. Geological Survey Water-Supply. 136pp.

Lancelle, H. G.; Longoni, C. A.; Ramos, A. O.; Caceres, J. R. (1986) Caracterización físico-química de ambientes acuáticos permanentes y temporarios del Chaco Oriental. Revista Ambiente Subtropical 73-91pp.

Ledesma, L. L.; Zurita, J. J. (1995) Los suelos de la Provincia del Chaco: Argentina, INTA (EERA Saenz Peña) Gobierno de la Provincia del Chaco. Report: 164pp.

Livingstone, D. A. (1963) Chemical composition of rivers and lakes. Data of geochemistry. US Geology Survey Prof. Paper 440 G: G1-G64pp. 
Ministerio de Salud y Ambiente de la Nación (MSAN), Organización Panamericana de la Salud (OPS), Prefectura Naval Argentina (PNA), Universidad Nacional de La Plata (UNLP) (2005) Caracterización sanitaria y ambiental de las aguas en tramos específicos de los Ríos Paraná y Paraguay y sus afluentes. Informe técnico: 9opp.

Morello, J. (1983) El gran Chaco: el proceso de expansión de la frontera agropecuaria desde el punto de vista ecológico ambiental. En: Expansión de la frontera agropecuaria y medio ambiente en América Latina. CEPAL-CIFCA, Argentina 10: 343-396pp.

Neiff, J.J. (1986) Sinópsis ecológica y estado actual del Chaco oriental. Revista Ambiente Subtropical 5-35pp.

Neiff, J. J.; Orfeo, O. (2003) Aporte de materia orgánica de los humedales a ríos de sabana subtropical del Chaco, Argentina. En Humedales de Iberoamérica: Argentina, Programa Iberoamericano de Ciencia y Tecnología para el Desarrollo: 142-152pp.

Neiff, J. J.; Orfeo, O. (2003) Aporte de materia orgánica de los humedales a ríos de sabana subtropical del Chaco, Argentina. En Humedales de Iberoamérica: Argentina, Programa Iberoamericano de Ciencia y Tecnología para el Desarrollo: 142-152pp.

Orfeo, O. (1999) Sedimentological characteristics of small rivers with loessic headwaters in the Chaco, South America. Quaternary International, 62: 69-74pp.

ORGANIZACIÓN DE LOS ESTADOS AMERICANOS (OEA), (1975) Estudio de la Cuenca Inferior del Río Bermejo. OEA-Gobierno Argentino; Tomo III, Recursos de la Tierra; 570pp.

Patiño, C.; Orfeo, O. (1986) Aproximación al conocimiento del proceso de erosión del suelo en el Chaco Oriental. Revista Ambiente Subtropical 47-59pp.

Patiño, C.; Orfeo, O. (1997) Cambios en la calidad del agua de los ríos Negro y Salado (Provincia del Chaco, Argentina), en relación con su régimen hidrológico y salinidad. Comunicaciones de la Secretaría General de Ciencia y Técnica. (último acceso 15/o8/2013).

Piper, A. M. (1944) A graphic procedure in the geochemical interpretation of water-analyses. Am. Geoph. Union Transv. V. 25, 914-923pp.

Poi de Neiff, A.; Patiño, C.; Neiff, J.J.; Ramos A. O. (2004) Calidad del agua en el tramo bajo del río Negro (Chaco, Argentina). FACENA Vol. 19: 67-85pp. 
Porta, J.; Lopez-Acevedo, M.; Roquero De Laburu, C. (1999) Edafología para la agricultura y el medio ambiente. $2^{\text {a }}$ Edición. Mundi Prensa (Ed.), España. 849pp.

RAMSAR (2013) (último acceso 12/11/2013).

http: / / www.ramsar.org/cda/es/ramsar-activities-world-wetlands-day-2004-19299/main/ramsar/1-63\%5E19299_4000_2__

Ribera, F.; Ordóñez, A. (2011) Curso Aspectos Hidrogeológicos de la Minería. Curso a distancia. a Fundación Centro Internacional de Hidrología Subterránea. Tema 3.

Ruberto, A. R. (1999) Hidroquímica de la cuenca del río Negro (Chaco). Tesis de Maestrando.

Sabater, S.; Elosegi, A. (2009) Presentación: importancia de los ríos. En: Elosegi, A. y Sabater, S. (Eds.): Conceptos y técnicas de ecología fluvial Fundación BBVA. España.

Smith, G.D. (1986) The Guy Smith Interviews: Rationale for concepts in Soil Taxonomy. SMSS Tech. Mon. $N^{\circ} 11$ SCS-USDA.

$\mathrm{ftp}: / / \mathrm{ftp}-\mathrm{fc} . \mathrm{sc}$. egov.usda.gov/NSSC/Soil_Taxonomy/Rationale.pdf (Última visita 07/06/2013)

Suárez, P.; Sartirana, M.; Orfeo, O. (2010) Caracteres sedimentológicos de ambientes fluviales de la llanura subtropical chaqueña (Argentina). Acta Geológica Lilloana, Vol. 22 (1): 34-45pp.

http://ubuntuone.com/1O7KpiC46vx1jwZD8429mz

Villar, C.A.; Bonetto, C. (2000) Chemistry and nutrient concentrations of the Lower Paraná River and its floodplain marshes during extreme flooding. Arch. Hydrobiology, 148: 3, 461-479pp.

Zhu, Y.; Lu, J.Y.; Liao, H. Z.; Wang, J. S.; Fan, B. L.; Yao S. M. (2008) Research on cohesive sediment erosion by flow: An overview. Science in China Series E: Technological Sciences. Springer. Vol. 51. No. 11. 